\title{
Concomitant Osteochondral Autograft Transplantation and Fixation of Osteochondral Fragment for Treatment of a Massive Osteochondritis Dissecans: A Report of 8-Year Follow-up Results
}

\author{
Byung Ill Lee, MD and Byoung Min Kim, MD \\ Department of Orthopedic Surgery, Soonchunhyang University Hospital, Seoul, Korea
}

\begin{abstract}
Numerous procedures exist to treat osteochondritis dissecans (OCD); however, it remains a topic of debate which procedure is most ideal. When restoring a massive osteochondral defect, the use of only one procedure may not always allow complete filling of the defect. This case report presents a massive OCD with displaced osteochondral fragment and loose body in the knee joint that occupied almost all of the weight bearing area of the medial femoral condyle and was treated with concomitant osteochondral autograft transplantation and fixation of displaced osteochondral fragment. To our knowledge, this is a rare report on OCD treated with concomitant osteochondral autograft transplantation and fixation of displaced osteochondral fragment. At 8 years after surgery, the clinical outcome was excellent, and radiographs revealed congruence of the medial femoral condyle. The patient returned to sports activities. In massive and complex OCD lesions, individual techniques have limitations. Two or more techniques are needed to increase the rate of success.
\end{abstract}

Keywords: Osteochondritis dissecans, Autologous transplantation, Internal fixation

Osteochondritis dissecans (OCD) is a localized condition in which a section of articular cartilage and underlying subchondral bone separate in the joint space. OCD, a condition of unclear etiology, most commonly occurs in the knees of adolescents and young adults. OCD lesions involve the medial femoral condyle in $85 \%$ of the cases and bilateral knees in $15 \%$ to $30 \%$ of the cases ${ }^{1)}$. If an OCD lesion involves the weight-bearing surface, it can induce pain at the knee joint and progress to degenerative joint diseases.

Numerous procedures exist to treat OCD; however, it remains

Received January 13, 2015; Revised May 6, 2015;

Accepted June 1, 2015

Correspondence to: Byoung Min Kim, MD

Department of Orthopedic Surgery, Soonchunhyang University

Hospital, 59 Daesagwan-ro, Yongsan-gu, Seoul 04401, Korea

Tel: +82-2-709-9253, Fax: +82-2-794-9414

E-mail: stompower@hanmail.net

This is an Open Access article distributed under the terms of the Creative Commons Attribution Non-Commercial License (http://creativecommons.org/licenses/by-nc/4.0/) which permits unrestricted non-commercial use, distribution, and reproduction in any medium, provided the original work is properly cited. a topic of debate which procedure is most ideal. Procedures for treating $\mathrm{OCD}$ are classified into two categories: repair techniques and restoration techniques. Repair techniques penetrate the subchondral bone and induce the formation of fibrocartilage tissue. Although excellent short-term clinical outcomes have been demonstrated after marrow stimulation ${ }^{2}$, long-term results of repair techniques have shown an objective and functional decline ${ }^{3)}$. Restoration techniques such as osteochondral autograft transplantation, mosaicplasty, and osteochondral allograft attempt to replace the cartilage defect with host or donor articular cartilage in a single stage. Clinical outcomes of osteochondral autograft transplantation have been reported to be good to excellent after seventeen years of follow-up in $>90 \%$ of patients with defects measuring 1 to $5 \mathrm{~cm}^{2,4)}$. Based on the extent of articular cartilage damage, the position of the lesion, and patient's age, surgeons can choose one of the treatment options. However, when restoring a massive osteochondral defect, the use of only one procedure may not allow complete filling of the defect.

Although reports on the treatment of massive OCD are common, reports regarding the use of concomitant osteochondral 
autograft transplantation and fixation of the displaced fragment are rare. Severity of arthroscopically assessed OCD lesions have been classified by the International Cartilage Repair Society into 4 types $^{5}$. Here, we describe a massive OCD with displaced osteochondral fragment and loose body in the knee joint, which occupied almost all of the weight bearing area of the medial femoral condyle. The patient was treated with concomitant osteochondral autograft transplantation and fixation of the displaced fragment, and we report 8-year follow-up results of the treatment.

\section{Case Report}

A 15-year-old boy had a 9-month history of left knee pain without trauma. The pain was aggravated during playing soccer one month prior to his presentation to this clinic. He presented with intermittent locking and popping of the knee but demonstrated a full range of motion (ROM). Initial radiographs revealed OCD of the medial femoral condyle (Fig. 1A, B). Preoperative computed tomography (CT) showed a large bony defect in the left medial femoral condyle. The size of the lesion was measured as $20 \times 33$ $\mathrm{mm}$ on CT (Fig. 1C). Magnetic resonance imaging (MRI) revealed a displaced fragment in the large crater and synovial fluid between the fragment and the underlying subchondral bone. A loose body was shown in the posteromedial pouch (Fig. 2).

On arthroscopy, displacement of an osteochondral fragment measuring $25 \times 20 \mathrm{~mm}$ in size was observed. In spite of reduction of the displaced osteochondral fragment in an appropriate position, an osteochondral defect measuring $8 \times 15 \mathrm{~mm}$ remained in the medial femoral condyle (Fig. 3A). A loose body measuring $10 \times 18 \mathrm{~mm}$ was found in the posteromedial compartment of the knee and removed.

The area of chondral separation was hinged on the intact posterior border of the lesion, revealing the fibrous bed (Fig. 3B). The crater was debrided to remove fibrous tissue, and microfracture was performed to promote blood supply to the base of the OCD fragment.

After the displaced fragment was reduced again, a guide pin was inserted, and headless compression screws (Acutrak Mini;
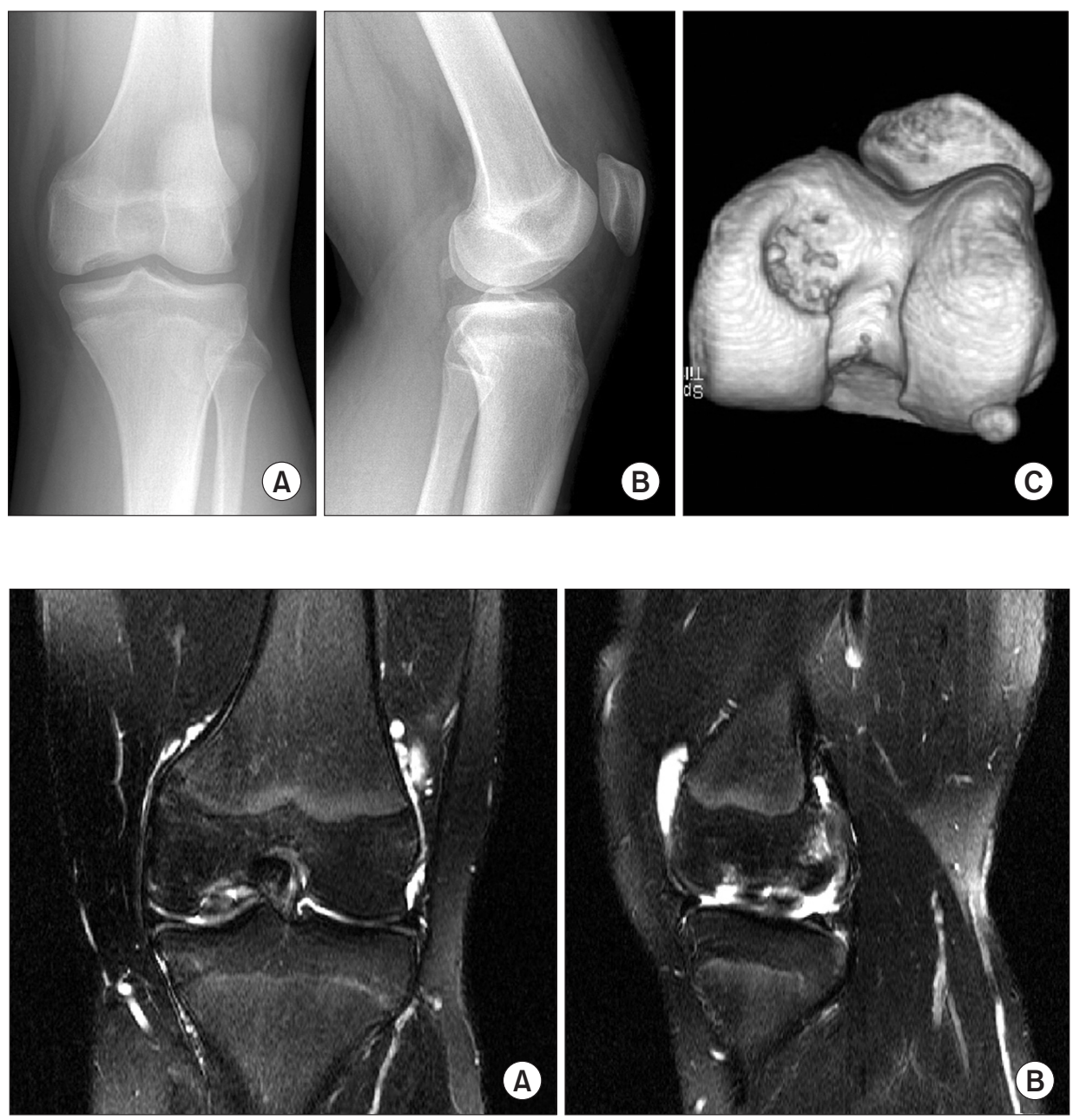

Fig. 2. (A) Coronal T2-weighted magnetic resonance imaging (MRI) showed a fluidfilled and detached osteochondral lesion. (B) Sagittal T2-weighted MRI showed detached and unstable fragments in the crater and the loose body. 

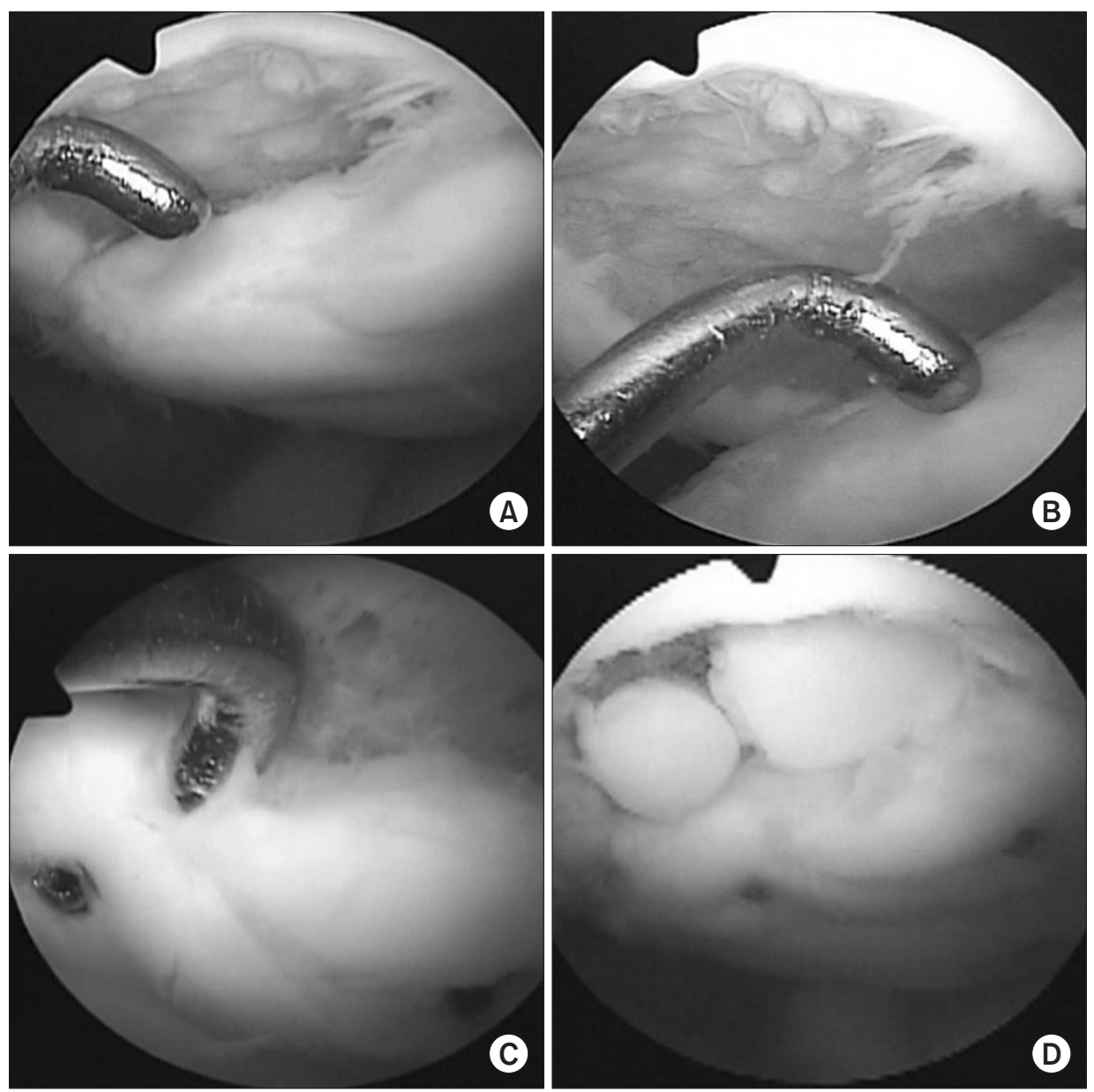

Fig. 3. Arthroscopic appearance of the osteochondral defect (A) and displaced osteochondral fragment (B). (C) After the displaced osteochondral fragment was fixed using a screw, the stability was confirmed. (D) Arthroscopic finding of the autogenous osteochondral graft in the defect lesion.

Acumed, Hillsboro, OR, USA) were inserted from the posterior aspect of the osteochondral lesion. Fixation was performed using four screws (Fig. 3C).

After fixation of the detached fragment, the size of the chondral defect decreased. Two bone plugs, $6 \mathrm{~mm}$ and $4 \mathrm{~mm}$ in diameter, were harvested from the intercondylar notch of the lateral condyle of the right knee using an osteochondral autograft transfer system (COR systems; Mitek, Norwood, MA, USA). Finally, the osteochondral grafts were transferred to the prepared defect site. All plugs were placed at the same level as the healthy cartilage (Fig. 3D). All procedures were performed by arthroscopy.

Progressive ROM was allowed from 1 week after surgery. The patient was then allowed to gradually increase ROM as tolerated. The patient was required to use crutches and an unrestricted ROM brace for 5 weeks and then allowed to increase weight bearing as tolerated at 6 weeks after surgery. The patient was pain-free with a full ROM and able to walk with the treated knee bearing full weight without any complications from 8 weeks after surgery.

At 1 year after surgery, arthroscopic screws were removed. Intraoperative findings revealed that the cartilage of the fixed fragment and osteochondral autograft were intact (Fig. 4A). The cartilage of the osteochondral lesion approximated well to the healthy surrounding cartilage (Fig. 4B).

The patient returned to sports activities. At 5 years after surgery, the follow-up MRI showed congruence of articular cartilage (Fig. $5 \mathrm{~A})$. The osteochondral plug had been completely incorporated (Fig. 5B). At 8 years after surgery, the patient provided informed consent for a report of his clinical results. The visual analog scale score with 10 representing the worst pain ever experienced improved from 9 preoperatively to 1 postoperatively. The Lysholm score increased to 95 from the preoperative score of 41 . The clinical outcome was excellent, and radiographs revealed congruence of the medial femoral condyle (Fig. 6). The patient had no swelling or locking of the right knee with full ROM.

\section{Discussion}

Numerous procedures exist for the treatment of OCD. However, it remains a topic of debate which treatment is most ideal. Excision of OCD fragments using the repair technique results in 

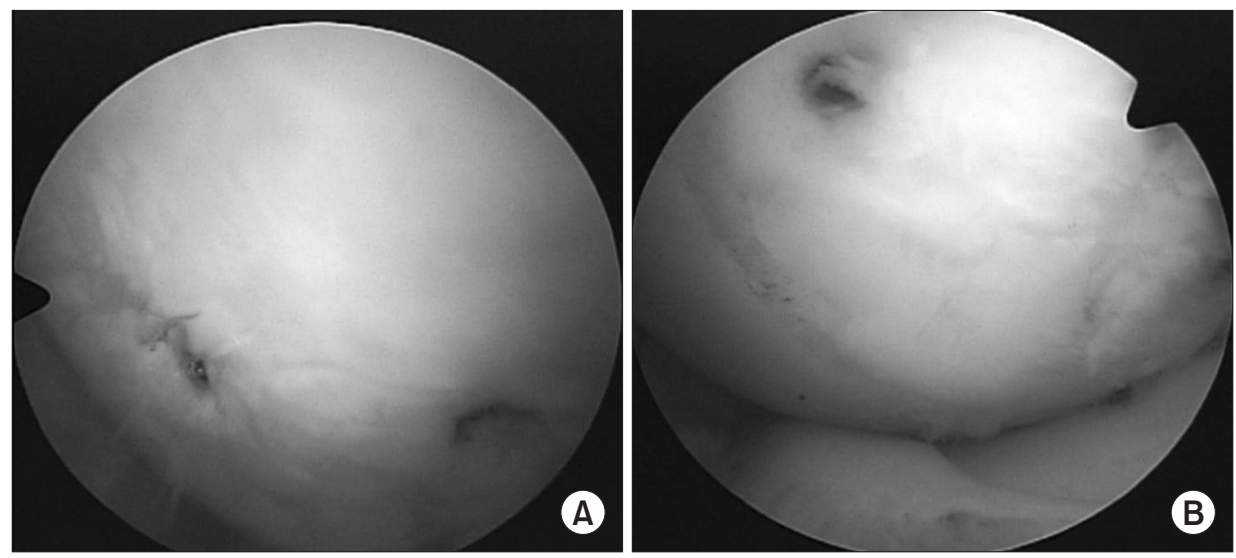

Fig. 4. (A) At 1 year after surgery, arthroscopy showed healed osteochondral graft. (B) After screws were removed, the osteochondral fragment was stable and well approximated to the healthy surrounding cartilage.
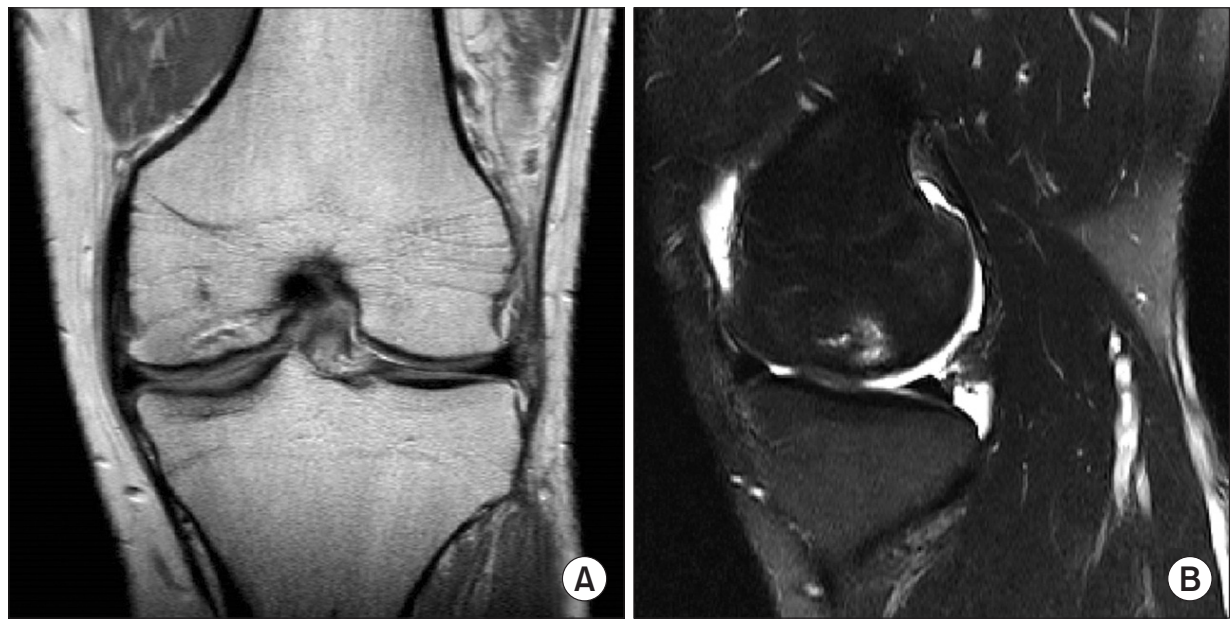

Fig. 5. Magnetic resonance imaging of the operated site in the medial femoral condyle at 5 years after surgery. (A) Coronal T2 proton dense image showed that congruence and continuity of cartilage was restored. (B) Sagittal T2-weighted image showed that the osteochondral plug had been completely incorporated. The signal intensity of the graft is homogenous with the surrounding subchondral bone, and the filling of the chondral defect is excellent with the transplanted cartilage.
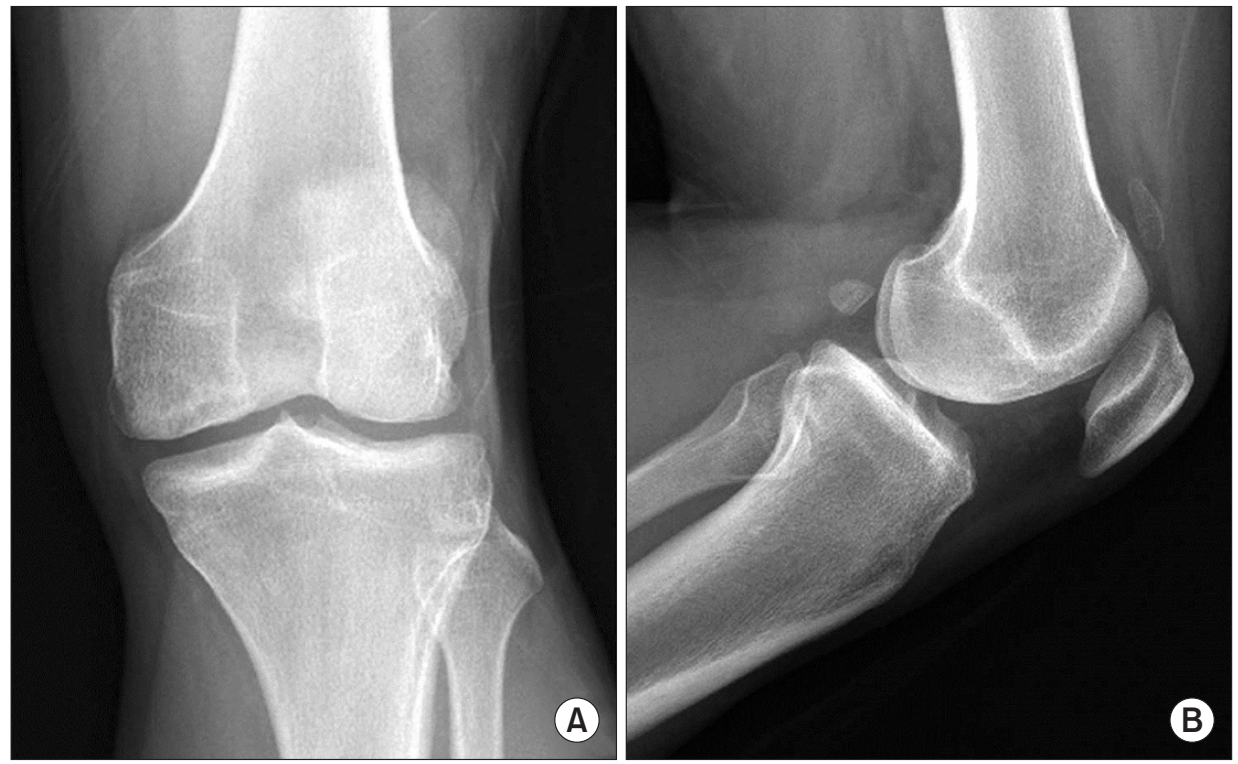

Fig. 6. Radiographs of the knee joint at 8 years after surgery. Anteroposterior view (A) and lateral view (B) radiographs of the knee joint revealed that the osteochondritis dissecans had healed while the joint maintained its congruity.

a high percentage of fair or poor outcomes ${ }^{6}$. Accordingly, efforts to preserve articular cartilage are necessary. Multiple principles should be applied in the surgical treatment of OCD lesions in the knee, including stable fixation, promotion of a blood supply to the base of the OCD fragment, and leveling of the cartilage of the OCD lesion to the articular margin of the healthy surrounding 
cartilage.

Our patient had a massive OCD lesion that was combined with an unstable osteochondral fragment with a loose body. Repair techniques, such as debridement and subchondral penetration, have been shown to have limited efficacy because of the poor biomechanical characteristics of the ingrown repair tissue. Previous multicenter, prospective studies have shown that mosaicplasty provides a substantially better clinical outcome than other repair techniques ${ }^{7)}$. However, donor site morbidity due to large osteochondral defects is an important limitation of osteochondral autograft transplantation. Due to the limitations for massive lesions or concurrent lesions of another type, concomitant techniques were necessary.

Loose body removal was performed in the present case. The displaced osteochondral flap was fixed after preparation of the crater base, since preservation of useful cartilage is essential for the success of the treatment. The osteochondral defect remained after fixation of the displaced fragment. Anderson and Pagnani ${ }^{3)}$ reported that loose-body removal and use of the repair technique produced short-term improvement that worsened with time. Guettler et $\mathrm{al}^{8}{ }^{8}$ reported a critical lesion size of $10 \mathrm{~mm}$ in diameter $\left(0.79 \mathrm{~cm}^{2}\right)$ over which defect rim stresses increased significantly. Jackson et al. ${ }^{9)}$ reported that full-thickness osteochondral defects in a goat model, measuring $6 \mathrm{~mm}$ in both diameter and depth, underwent progressive changes. If the osteochondral defect was treated by drilling or microfracture, the lesion could result in additional stress on the fixed osteochondral fragment and may hinder the healing of the osteochondral fragment. Therefore, for the defect lesion, osteochondral autograft transplantation was performed to prevent collapse of the fixed osteochondral fragment and to protect the surrounding cartilage and the fixed osteochondral fragment from additional stresses. Consequentially, the fixation of the osteochondral fragment could reduce donor site morbidity in osteochondral autograft transplantation because of a smaller harvest, and the osteochondral autograft transplantation could protect the fixed osteochondral fragment from additional collapse.

The combination of autologous chondrocyte implantation and osteochondral graft for massive osteochondral defect has been used for reconstruction of the joint surface with excellent clinical outcomes $^{10)}$. To our knowledge, reports on the use of concomitant osteochondral autograft transplantation and fixation of displaced fragment in massive OCD lesion with 8-year follow-up are rare.

For the treatment of massive and complex OCD lesions, individual technique has some limitations. Two or more techniques are needed to increase the rate of success. Therefore, preservation of useful cartilage is essential for successful treatment and additional procedures should be considered to restore the congruence of the cartilage in the knees with massive or complex OCD lesions.

\section{Conflict of Interest}

No potential conflict of interest relevant to this article was reported.

\section{Acknowledgements}

This work was supported by Soonchunhyang University Foundation of Korea.

\section{References}

1. Crawford DC, Safran MR. Osteochondritis dissecans of the knee. J Am Acad Orthop Surg. 2006;14:90-100.

2. Mithoefer K, McAdams T, Williams RJ, Kreuz PC, Mandelbaum BR. Clinical efficacy of the microfracture technique for articular cartilage repair in the knee: an evidence-based systematic analysis. Am J Sports Med. 2009;37:2053-63.

3. Anderson AF, Pagnani MJ. Osteochondritis dissecans of the femoral condyles. Long-term results of excision of the fragment. Am J Sports Med. 1997;25:830-4.

4. Hangody L, Dobos J, Balo E, Panics G, Hangody LR, Berkes I. Clinical experiences with autologous osteochondral mosaicplasty in an athletic population: a 17-year prospective multicenter study. Am J Sports Med. 2010;38:1125-33.

5. Brittberg M, Winalski CS. Evaluation of cartilage injuries and repair. J Bone Joint Surg Am. 2003;85 Suppl 2:58-69.

6. Wright RW, McLean M, Matava MJ, Shively RA. Osteochondritis dissecans of the knee: long-term results of excision of the fragment. Clin Orthop Relat Res. 2004;(424):239-43.

7. Gudas R, Kalesinskas RJ, Kimtys V, Stankevicius E, Toliusis V, Bernotavicius G, Smailys A. A prospective randomized clinical study of mosaic osteochondral autologous transplantation versus microfracture for the treatment of osteochondral defects in the knee joint in young athletes. Arthroscopy. 2005;21:1066-75.

8. Guettler JH, Demetropoulos CK, Yang KH, Jurist KA. Osteochondral defects in the human knee: influence of defect size on cartilage rim stress and load redistribution to surrounding cartilage. Am J Sports Med. 2004;32:1451-8. 
9. Jackson DW, Lalor PA, Aberman HM, Simon TM. Spontaneous repair of full-thickness defects of articular cartilage in a goat model: a preliminary study. J Bone Joint Surg Am. 2001;83:53-64.

10. Gudas R, Simonaityte R, Cekanauskas E, Mickevicius T.
Concomitant autologous chondrocyte implantation with osteochondral grafting for treatment of a massive osteochondral defect in the bilateral knees of a child. Medicina (Kaunas). 2011;47:170-3. 